\title{
Turismo extranjero y crecimiento económico en México. Evidencia empírica para Los Cabos
}

\section{Foreign tourism and economic growth in Mexico. Empirical evidence for Los Cabos}

\author{
Gustavo R. Cruz-Chávez* \\ Víctor Hernández-Trejo* \\ Gerzaín Avilés-Polanco* \\ Ramón Valdivia-Alcalá ** $^{*}$
}

\begin{abstract}
Tourism in Mexico is important because it generates economically significant effects on growth. Using quarterly data on international tourist arrivals to Los Cabos (AT), the real exchange rate (RER) and gross domestic product (GDP) in Mexico, we apply co-integration analysis to demonstrate two aspects: a) at least one co-integration vector between domestic product (GDP) in Mexico and the RER and at, we obtain inelastic elasticities for both variables (positive and negative, respectively); and, b) a long-run stable relationship between GDP and AT.
\end{abstract}

Keywords: Tourism growth, Los Cabos, co-integration, tourism policy in Mexico

\section{Resumen}

El turismo en México es significativo económicamente por generar importantes efectos en el crecimiento. En esta investigación se utilizan datos trimestrales de arribos turísticos (AT) internacionales a Los Cabos, el tipo de cambio real (TCR) y el Producto Interno Bruto (PIB) de México para hacer un análisis de cointegración que demuestra dos aspectos: $a$ ) al menos un vector de cointegración entre el pIB de México, el TCR y los AT, obteniendo para ambas variables elasticidades inelásticas (positiva y negativa, respectivamente) y, $b$ ) una relación estable de largo plazo entre el PIB y los AT.

Palabras clave: Turismo y crecimiento, Los Cabos, cointegración, política turística en México

\footnotetext{
*Universidad Autónoma de Baja California Sur. correo-e: gcruz@uabc.mx, victorh@uabcs.mx, gaviles@uabcs.mx, gcruz@uabcs.mx

** Universidad Autónoma Chapingo. Correo-e: ramval@correo.chapingo.mx
} 


\section{Introducción}

El análisis de la relación entre el flujo turístico internacional al centro integralmente planificado, Los Cabos, y el crecimiento económico de México, medido a través del PIB, se realiza con base en la idea propuesta por Brida et al., (2008a). La United World Tourism Organization (UNWTO, 2011) ubicó a México, hasta el 2011, como el décimo destino más importante en afluencia turística internacional; actualmente ocupa el lugar número 15 (UnwTo, 2014). A nivel de entidad federativa Tello-Contreras et al. (2012) ubica a Baja California Sur como el segundo destino a nivel nacional.

De acuerdo con datos de la Subsecretaria de Planeación Turística (2012), Los Cabos es el segundo centro integralmente planeado (CIP) con más visitas al año (después de Cancún) y el cuarto destino como centro de playa. A pesar de estas cifras, existen pocos estudios sobre el efecto económico del turismo internacional sobre el рів de México. En el marco de esta temática, los primeros estudios del turismo, como determinante del crecimiento económico en México, inician con Ramírez-Hernández (2006), quien incorpora el PIB nacional, el PIB de Estados Unidos y el PIB turístico en México, construido a partir de 12 variables reportadas en los censos económicos.

Otros dos estudios a nivel macroeconómico son los realizados por Brida et al. (2008a) y De la Cruz-Gallegos et al. (2010); el primero incorpora en su análisis al PIB nacional, tipo de cambio real y gasto del turismo; el segundo realiza un análisis que toma como variables el arribo de turistas y el PIB mexicano. Sin embargo, no existen estudios a nivel de entidad federativa, local o por polo de desarrollo, tampoco se han realizado estudios sobre los efectos de la política de desarrollo turístico planteada por el gobierno federal en la década de los 70 para impulsar algunos polos de desarrollo turístico en el territorio nacional.

El periodo de análisis se ubica entre 1995 y 2011, el primer año corresponde a un cambio estructural en la macroeconomía nacional, el último es de interés para el estudio, ya que la UNWTO pronostica que para ese año ya se podrá ver una recuperación en el flujo turístico internacional a México, sin mencionar que también para la actividad turística de las entidades federativas.

Los datos utilizados corresponden a series de tiempo trimestrales del PIB nacional, afluencia turística internacional al centro integralmente planeado Los Cabos y tipo de cambio real (TCR). Los objetivos de la investigación son: i) comprobar la existencia de una relación estable a largo plazo entre el рів y la afluencia turística, ii) comprobar la hipótesis de crecimiento impulsada por el turismo para este polo de desarrollo turís- 
tico y, iii) evaluar el crecimiento económico de largo plazo en México, derivado de la política de desarrollo turístico planteada en la década de los setenta. Para lograr esto se utiliza el análisis de cointegración de Engle y Granger y el método de Johansen y Joselius.

\section{Panorama mundial del turismo}

La United Nations World Tourism Organization (UNwTO, 2011) menciona que tras haber pasado por una crisis intensa por el brote del AH1N1 y la crisis financiera internacional, para el primer semestre de 2010 la actividad turística global muestra signos de recuperación. En cifras más actuales, la UNwTo (2013) reporta que para finales del 2012 se ve una recuperación del turismo mundial, posterior a la crisis iniciada en 2007; asimismo, los efectos del AH1N1 se ven desvanecidos, ya que las llegadas de turistas internacionales crecieron $5 \%$ en los primeros nueve meses del año, alcanzando la cifra récord de 845 millones en todo el mundo: 41 millones más que en el mismo periodo de 2012.

El crecimiento fue impulsado por Europa y Asia-Pacífico, donde el número de turistas aumentó $6 \%$ en ambas regiones. El turismo internacional aumentó aún por encima de las expectativas, contribuyendo al crecimiento económico en las economías avanzadas y emergentes. La recuperación se puede observar de forma más clara en destinos europeos, donde el turismo ha sido un importante elemento para la recuperación económica.

El crecimiento de las llegadas internacionales y el ingreso por turismo a nivel mundial alcanzó 5\% en 2013, con 1,087 millones de turistas (+52 millones que en 2012) y 1,159 millones de dólares (+81 millones que en 2012). México dejó de pertenecer al selecto grupo de los 10 principales destinos por llegadas internacionales, pasando el lugar número 15 en 2013, superado por economías emergentes como Tailandia y Rusia.

En el mismo documento, la UNwTO (2013) menciona que según las previsiones a largo plazo, las llegadas de turistas internacionales a escala mundial crecerán 3.3\% anualmente entre 2010 y 2030, hasta alcanzar los 1.800 millones. Se prevé que el ritmo de crecimiento de llegadas en destinos emergentes $(+4.4 \% \mathrm{al}$ año) doble el de las economías avanzadas $(+2.2 \%$ al año). La cuota de mercado de las economías emergentes ha aumentado de $30 \%$, en 1980 , a $47 \%$, en 2013 ; se prevé que alcance $57 \%$ en 2030 , lo que equivale a más de mil millones de llegadas de turistas internacionales.

El wTTC (2013a) indica que el impacto del turismo en el pIB mundial aumentó por cuarto año consecutivo a 9.5\% (7 trillones de dólares); se crearon aproximadamente 4.7 millones de nuevos empleos alrededor del 
mundo como resultado de la actividad turística del año pasado. Actualmente, el sector mantiene cerca de 266 millones de personas empleadas, lo que significa uno de cada 11 empleos a nivel global. Para 2014 se pronosticó un incremento de $4.3 \%$, el cual se debería al gasto turístico como recuperación de la recesión global; se esperaban 6.5 millones de nuevos empleos para el mismo año.

\subsection{Perspectiva del turismo en México}

La actividad turística en México muestra signos de recuperación de los embates de la crisis financiera y del fenómeno AH1N1. La unwTo (2013) menciona que la Región de América del Norte capta cerca de dos tercios del todas las llegadas del continente americano, registrando un aumento de 3\%. Los Estados Unidos de norteamérica registraron un sólido incremento de llegadas de turistas equivalente a 5\%, mientras que Canadá registró 2\% y México 1 por ciento.

El wTTC (2013b) indica que para el 2013 el turismo en México contribuyó directamente al PIB con $5.9 \%$ (+0.3 con respecto al ańo anterior), alrededor de 953 mil millones de pesos, y de forma agregada con el 13.3\% (+2.1 con respecto a 2012). Se generaron 3.180 millones de empleos directos $(6.4 \%$ del total) y de forma agregada (incluyendo empleos indirectos) 7.0235 millones de los empleos (14.2\% del total).

En lo que respecta a la inversión turística, en México fue de alrededor 306 mil millones de pesos, lo que representó $8.9 \%$ de la inversión total. Conforme a la importancia relativa de la contribución total de los viajes y turismo al рів, México se ubica, de entre 184 países, en la posición nueve con respecto al tamaño; en el 57 con respecto al tamańo relativo; en el 98 en lo relacionado al crecimiento, y ocupa el lugar 78 en cuanto a crecimiento a largo plazo. Por otra parte la UNwTo (2014) menciona que México dejó de pertenecer al grupo de los 10 principales destinos por llegadas internacionales, como se mencionó anteriormente, se colocó en el sitio 15 en 2013, superado por economías emergentes como Tailandia y Rusia.

\subsection{Turismo en Baja California Sur}

En lo concerniente a Baja California Sur (BCS), el turismo ha sido desde hace más de cinco décadas motor de la actividad económica en el estado. El Sistema Integral de Información de Mercados Turísticos (sIImT, 2012) reporta que a nivel nacional BCS se ubica en la posición 19 en número de visitantes. En 2010, el sector terciario contribuyó con 77\% al PIB estatal (53.09 millones de pesos). En lo concerniente al empleo, el Instituto 
Nacional de Estadística Geografía e Informática (INEGI, 2012a) reporta que el turismo aporta $9 \%$ de los empleos directos y $2 \%$ de los indirectos.

Para el periodo de 2003 a 2010, el sector terciario participó en promedio con $78 \%$ al PIB estatal, con alrededor de 36 millones de pesos en promedio y un crecimiento interanual promedio de 4 por ciento. Según datos del siımt (2012), el turismo en BCs disminuyó entre 2007 y 2008 alrededor de 7\%; mientras que entre 2007 y 2010 esta variable se redujo en más de 22\%, lo cual se atribuye al efecto del AH1N1 y la crisis financiera internacional.

\subsection{Turismo en el centro integralmente planificado Los Cabos}

De acuerdo con Magaña-Carrillo (2009) y Molina (2008), la política de desarrollo turístico que dio origen a los Centros integralmente planeados (CIP) tuvo lugar en la década de los setenta, ésta se sostuvo en acciones y lineamientos que contribuyeron a aumentar la afluencia de visitantes. Se impulsaron sitios mediante estrategias de planeación turística para su desarrollo, constituyendo una ventaja en cuanto al aprovechamiento de los recursos y la derrama de beneficios regionales, lo cual fortaleció al sector turístico. En ésta década, la Secretaría de Turismo (Sectur) y el Fondo Nacional de Turismo (Fonatur) fungen como instituciones encargadas de la planeación y fomento de la actividad turística para su desarrollo.

La estrategia más destacada es la creación del Plan maestro de turismo para México, en él se formula la creación de cinco CIP en torno a cuatro de los estados más pobres de México que tienen grandes extensiones de costa: Cancún (Quintana Roo), Ixtapa-Zihuatanejo (Guerrero), Bahías de Huatulco (Oaxaca), y Los Cabos y Loreto (Baja California Sur), todos ellos se inician en el primer lustro de la década.

En sus inicios, entre 1970 y 1980, las acciones de política pública de desarrollo turístico en el cip Los Cabos estuvieron encaminadas a la creación de infraestructura de apoyo para el turismo, principalmente comunicación (construcción de la carretera federal México 1 o carretera transpeninsular, rutas de transbordador y la puesta en operación de un aeródromo y pistas de aterrizaje), el gobierno federal y estatal realizan inversiones en materia de infraestructura urbana (pavimentación, alcantarillado y agua potable, así como iluminación y diseño arquitectónico).

Para la siguiente década, 1980-1990, se da prioridad a la conectividad aérea del destino y se construye el aeropuerto internacional de San José del Cabo; Fonatur continua realizando inversiones en infraestructura e imagen. En este mismo periodo la afluencia turística internacional tiene un aumento originado por la devaluación de 1982 y otras subsecuentes. 
De 1990 al 2000 es el periodo de consolidación; el eclipse total de sol de 1991 es el parteaguas que coloca a Los Cabos en el panorama mundial; la afluencia turística extranjera incrementa notablemente debido a una intensa campaña mediática de promoción. Asimismo, la federación y el gobierno del estado continúan ampliando la infraestructura (se construye el acueducto y la carretera cuatro carriles San José del Cabo-Cabo San Lucas, también se amplía el aeropuerto de San José del Cabo).

En la última década del periodo 2000-2010, se realiza en Los Cabos la reunión de la APEC (Asia-Pacific Economic Cooperation) logrando que el destino sea promovido en todo el mundo, al mismo tiempo, se comienzan acciones para la ampliación de dos terminales aéreas para el aeropuerto de San José del Cabo. La inversión acumulada desde 1999 hasta 2009 por parte de Fonatur en Los Cabos alcanza un valor agregado de casi 583 millones de pesos (Gobierno del Estado de Baja California Sur, 2009).

Todas estas acciones de política de desarrollo e inversión, unidas a eventos de gran relevancia internacional, lograron posicionar a Los Cabos como un destino internacional importante; sin embargo, el crecimiento sostenido que había mostrado Los Cabos en las últimas dos décadas se ve frenado por los efectos de la crisis financiera internacional de 2008 y por el AH1N1.

Por otra parte, se considera que el turismo, principalmente extranjero, ocasiona una dinámica de arrastre en la economía de Los Cabos. De acuerdo con resultados definitivos de los censos económicos 2009 que reporta el INEGI (2012b), las principales actividades que concentran alrededor de $72 \%$ de los ingresos en orden descendente son: $a$ ) comercio al por menor, con $30.0 \%$; b) servicios de alojamiento temporal y de preparación de alimentos y bebidas, con $29.2 \%$; c) comercio al por mayor, con 12.8 por ciento.

Otras actividades relevantes en cuanto a su participación proporcional en los ingresos del municipio son la construcción (7.1\%) y los servicios de apoyo a los negocios, manejo de desechos y de remediación (4.6\%). Estas cinco actividades son el soporte de la economía de Los Cabos y suman $85 \%$ de los ingresos municipales. Debido a lo anterior se considera al turismo en Los Cabos la actividad que sostiene la economía local; de ella depende aproximadamente $82 \%$ de la población, ya sea directa o indirectamente.

La afluencia turística entre 1976 y 2011 en el cip Los Cabos ha mostrado una tendencia creciente, a pesar de momentos económicos adversos como crisis, devaluaciones y eventos coyunturales. Los Cabos se ha consolidado como polo turístico de desarrollo económico para la zona.

En la gráfica 1 se puede apreciar la tendencia creciente del flujo turístico extranjero, al igual que descensos severos en este flujo para 1981, 
1985, 1998 y 2009; estos elementos coyunturales macroeconómicos que afectan la afluencia turística extranjera al cip Los Cabos no han podido, al parecer, detener la tendencia incremental de la misma. Los visitantes al CIP Los Cabos tienen un gasto promedio de entre 1,801 y 1,917 dólares (Billfish Foundation, 2008 y Ditton et al., 1996), que es mayor en comparación a la media nacional de 972 dólares (sinmt, 2012).

Entre 1976 y 2011 la tasa de crecimiento media anual de la afluencia turística total al cIp Los Cabos fue de 70\%; de forma desagregada el turismo nacional e internacional crecieron a una tasa media anual de $45 \mathrm{y}$ 89\%, respectivamente. Se observa un crecimiento sostenido hasta el 2007. Es a partir de 2008 cuando se comienza a ver un descenso en la afluencia turística internacional del cip Los Cabos, lo cual se atribuye a los efectos impacto de la crisis financiera mundial ocurrida entre 2008 y 2009 y a los del virus $\mathrm{AH} 1 \mathrm{~N} 1$. El flujo de turistas extranjeros muestra una ligera recuperación para el 2010.

\section{Gráfica 1}

Afluencia turística extranjera en el CIP Los Cabos, 1976-2011

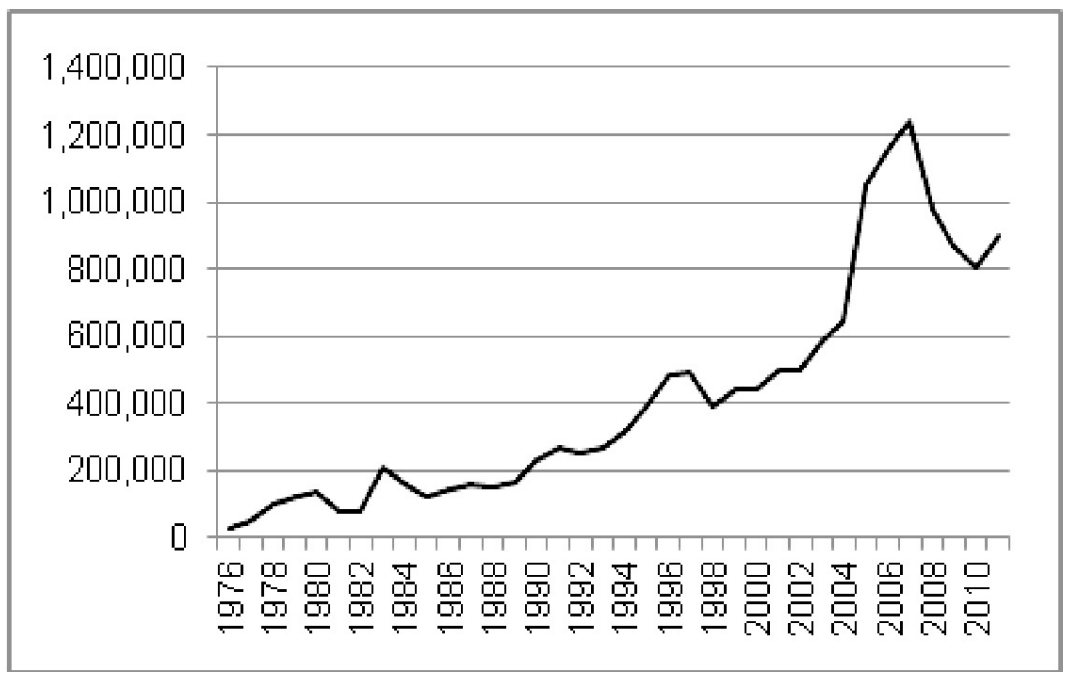

Fuente: elaboración propia.

Para el 2011, Los Cabos recibió 1.237 millones de visitantes, cuya distribución proporcional es $73 \%$ extranjeros y $27 \%$ turistas nacionales. En el periodo poscrisis, entre el 2007 y el 2011, Los Cabos muestra tasa de crecimiento media anual negativa de $0.1 \%$, lo cual se toma como indicador de recuperación del destino de los efectos que dejó la crisis financiera. Los vuelos operados en el 2009 en el Aeropuerto internacional de San José del Cabo fueron 13,894, con un total de arribos registrados que 
ascienden a 2.655 millones de pasajeros (31\% arribos nacionales y $69 \%$ internacionales). En cuanto al turismo que arriba vía marítima, en el mismo año se tuvieron 288 cruceros que transportaban 665,477 pasajeros.

\section{La hipótesis de crecimiento impulsada por el turismo}

Balaguer y Cantavella-Jordá (2002) fueron los primeros autores en introducir el concepto de la hipótesis del crecimiento económico impulsado por el turismo $\left(\mathrm{TLGH}^{\mathrm{I}}\right.$, por sus siglas en inglés). El interés de estos autores en medir el impacto del turismo al crecimiento económico surge de los pesos relativos de esta actividad en el PIB de los principales países receptores, como Estados Unidos, que ocupa el segundo lugar en recepción de turistas internacionales y el primero en ingreso de divisas, no obstante, la participación del sector en el PIB, que representa sólo 1.9\%, mientras que España ocupa el cuarto lugar en arribos y el segundo en ingreso de divisas por turismo internacional, lo cual representa 5.9\% de su PIB.

En este sentido, Balaguer y Cantavella-Jordá (2002) encontraron una relación estable de largo plazo de la afluencia de turismo internacional y el pIB español. Este estudio motivó un rápido crecimiento de la bibliografía empírica sobre el turismo como determinante del crecimiento económico (gráfica 2).

\section{Gráfica 2}

\section{Evolución de estudios sobre la TLGH ${ }^{2}$}

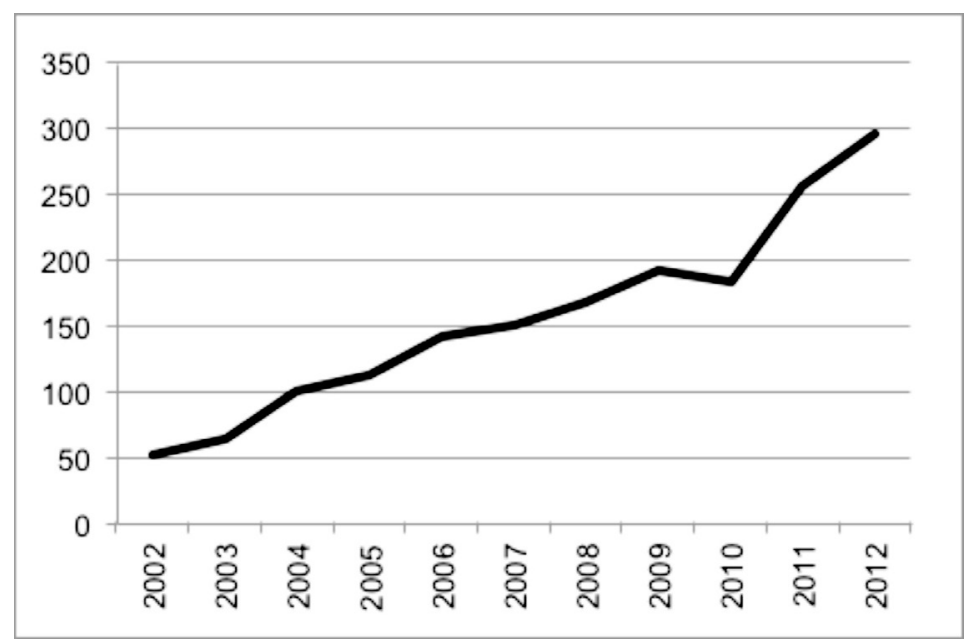

Fuente: elaboración propia

\footnotetext{
${ }^{1}$ Tourism Led Growth Hypothesis.

${ }^{2}$ Incluye las palabras Tourism Growth y TLGH.
} 
Existen 22 estudios que confirman los resultados obtenidos por Balaguer y Cantavella-Jordá (2002) (anexo 1), los cuales indican una relación causal entre el PIB y el arribo de turistas extranjeros, como el de Gunduz y Hatemi-J (2005) para Turquía. Sin embargo, la reciente literatura no ha consensado la relación de causalidad, ya que 11 de ellos hallan una relación bidireccional entre ambas variables (Kim et al., 2006; Ongan y Demiroz, 2005 y Dritsakis, 2004) implicando que una expansión en el turismo internacional estimula el crecimiento de la actividad económica y viceversa (anexo 2).

Por otra parte, existen nueve estudios que no reportan relación causal en el sentido de Granger entre el pib y la afluencia turística (anexo 3), es decir, no se encuentra evidencia que valide el cumplimiento de la TLGH (Cortés-Jiménez y Pulina, 2006; Oh, 2005). Las inconsistencias de los resultados en diversos países han puesto en el debate a la TLGH. La mayoría de estos estudios han utilizado el PIB, los arribos de turistas internacionales y el gasto medio de turistas internacionales, así como el tipo de cambio real. En este sentido, Balaguer y Cantavella-Jordá (2002) sugieren que esta última variable debe ser incluida en la discusión empírica.

La participación del sector turismo en la economía de muchos países y su aportación al crecimiento económico de largo plazo, sobre todo en países menos desarrollados, atrajo la atención de investigadores que buscan analizar esta relación. Este interés ha propiciado que la investigación de la economía del turismo sea el principal tema de la literatura empírica reciente de apenas una década, investigación que busca probar la existencia de causalidad entre el crecimiento medido a través de variables como el PIB y variables a proxy tales como el PIB per cápita (Brida et al., 2011), la producción industrial (Lean y Tang, 2010), el índice de producción industrial (Tang, 2011), la inversión extranjera directa (Tang y Hang, 2009) y el volumen de comercio internacional, así como el ingreso disponible (Khan et al., 2005).

\section{La Tourism Led Growth Hypothesis (TLGH) en México}

Brida et al. (2008a) confirman el cumplimento de TLGH en México, encuentran causalidad entre el gasto de turismo, el tipo de cambio real y el PIB mexicano, con frecuencia trimestral, de 1980 a 2007. Los autores emplean un enfoque econométrico consistente en contrastes de cointegración, como el método de Johansen (1988) y el método de Johansen y Joselius (1990); reportan la existencia de un vector único de cointegración y concluyen existencia de causalidad unidireccional del gasto del turismo y tipo de cambio real al PIB nacional. 
Un aspecto importante en torno al hallazgo de Brida et al. (2008a) consiste en el signo del coeficiente del tipo de cambio real, que resultó positivo; este resultado no es consistente con la literatura empírica, ya que Kamin y Rogers (1997) argumentan que devaluaciones reales se encuentran asociadas a procesos inflacionarios altos y a contracciones de la actividad económica. En este sentido, Galindo y Guerrero (2001), en un estudio que realizan sobre los efectos de la recesión de Estados Unidos y del tipo de cambio real sobre el crecimiento económico en México, encuentran una asociación negativa del tipo de cambio real sobre el crecimiento económico de largo plazo en México. Por su parte, Garcés-Díaz (2006) argumenta que el efecto del tipo de cambio real es positivo sobre la balanza comercial, pero negativo para el resto de la economía, lo cual es consistente con Galindo y Guerrero (1997) y López y Guerrero (1998), al cumplirse la condición Marshall-Lerner en México, ya que una depreciación del tipo de cambio real mejora el saldo externo. Recientemente De la Cruz-Gallegos et al. (2010) contrastaron el cumplimiento de la TLGH en México mediante un modelo de Corrección del Error en Forma Vectorial (VECM, por sus siglas en inglés), encontrando una relación de causalidad positiva entre el arribo de turistas y el PIB mexicano; sugiriendo que la TLGH se cumple en México a nivel macroeconómico.

\section{Materiales y métodos}

El marco temporal de análisis del presente trabajo se ubica entre los años 1995 y 2011. En 1995, México atravesó por una crisis originada por una devaluación del tipo de cambio real peso/dólar. A partir de ese año la economía mexicana registró un crecimiento económico sostenido hasta el 2008, fecha en la que es posible apreciar una contracción simultánea del PIB mexicano y aT en el CIP Los Cabos.

Para la primera variable esta contracción se puede atribuir a la crisis financiera originada por la burbuja financiera en el sector inmobiliario de los Estados Unidos de Norteamérica, para la segunda, el descenso se adjudica a los efectos de la crisis financiera internacional aunado a los efectos del virus AH1N1.

Es importante resaltar que el descenso registrado en la afluencia de turistas internacionales en Los Cabos se asocia al impacto negativo de la recesión económica sobre los turistas norteamericanos, ya que éstos constituyen el principal origen de los turistas internacionales a este destino. El periodo de estudio finaliza en el 2011, ya que en este ańo se manifiesta una recuperación de los efectos de ambos fenómenos.

El pIB fue obtenido del Banco de Información Económica (BIE) del INEgi (2010), la afluencia turística extranjera a Los Cabos se obtuvo del 
SIIMT (2012), el tipo de cambio real bilateral fue obtenido del Centro de Estudios de Finanzas Públicas del H. Congreso de la Unión (2010). Todas las variables se encuentran con frecuencia a partir de 1995 y hasta el 2011.

Para comprobar la TLGH en el CIP Los Cabos se utilizó la metodología de cointegración, que permite identificar relaciones estables de largo plazo entre variables de interés (Quintana-Romero y Mendoza-González, 2008; Castillo-Ponce y Varela-Llamas, 2010). La cointegración menciona que si se consideran dos procesos estocásticos integrados de orden uno: $y_{t} \sim I(1)$ y $x_{t} \sim I(1)$. En general, una combinación lineal de estas variables se esperaría estuviera integrada de orden 1 esto es $y_{t}-p_{I} x_{t}=\varepsilon_{\mathrm{t}}-\mathrm{I}(1)$.

Sin embargo, en algunas instancias las series $y_{t}$ y $x_{t}$ pueden compartir una tendencia estocástica común; de tal forma que la combinación lineal de las series podría estar integrada de orden cero, o ser estacionaria, formalmente tendríamos $y_{t}-p_{t} x_{t}=\varepsilon_{t} \sim I(0)$. En dado caso, se dice que $y t$ y $x t$ están cointegradas. La definición de cointegración que se presenta no es la que comúnmente se describe en libros de texto, que se refiere a la propiedad que exhiben series de tiempo "moverse juntas". Esta frase, que originalmente postularan Engle y Granger (1987), se considera que ejemplifica apropiadamente el caso en el que las series se asocian positiva o negativamente; así que el concepto de cointegración se refiere más puntualmente a la propiedad que exhiben series de tiempo de compartir tendencia común.

A partir de esa caracterización es posible determinar los coeficientes de cointegración, los cuales, de hecho, representan los ponderadores que hacen posible que la combinación lineal de $y_{\mathrm{t}}$ y $x_{\mathrm{t}}$ sea estacionaria. La instrumentación del ejercicio de cointegración se puede llevar a cabo bajo diferentes metodologías, como el método de Engle y Granger y el método de Johansen y Joselius. A continuación se describen estos métodos.

El método de dos etapas sugerido por Engle y Granger (1987) consiste en instrumentar una estimación bietápica que prueba la idea fundamental de cointegración, que consiste en que una combinación lineal de series no-estacionarias es en sí misma estacionaria. Para introducir la metodología, a continuación se ilustra un caso bivariado. Es necesario considerar dos series integradas de orden 1, $y_{t} \sim I(1)$ y $x_{t} \sim I(1)$. Es posible representar una ecuación de regresión entre las mismas como sigue

$$
y_{t}=y x_{t}+\varepsilon_{1}
$$

Donde $\varepsilon_{1}$, representa el término de error que se supone es ruido blanco. 
La primera etapa de la metodología consiste en estimar la ecuación (1) por medio de mínimos cuadrados ordinarios y obtener los errores estimados, $\boldsymbol{\varepsilon}_{t}$. En la segunda etapa se realiza una prueba de estacionariedad para $\varepsilon_{t}$; si el término de error es estacionario, entonces se concluye que $y_{t}$ y $x_{t}$ se encuentran cointegradas, y la elasticidad de largo plazo de $y_{t}$ con respecto a $x_{t}$, es el coeficiente $y$. Si por el contrario, $\varepsilon_{t}$; resulta noestacionario entonces se concluye que $y_{t}$ y $x_{t}$ no comparten una tendencia común y, por ende, los resultados de la primera etapa no son confiables, en otras palabras, la relación entre $y_{t} \mathrm{y} x_{t}$ es espuria.

La ecuación (1) se puede reescribir como $y_{t}-y x_{t}=\varepsilon_{t}$, de tal forma que $\varepsilon_{t}$, representa una combinación lineal de $y_{t} y x_{t}$, así que al estimar $\varepsilon_{t}$, y realizar la prueba de estacionareidad sobre la serie, se está verificando el concepto mismo de cointegración, la existencia de una combinación lineal de $y_{t}$ y $x_{t}$ que es estacionaria. Cabe destacar que para realizar la prueba de estacionareidad sobre $\varepsilon_{t}$.

El método de Johansen y Joselius (1990) consiste en aplicar un modelo de corrección de error mediante la ecuación siguiente $\Delta Z_{t}=\Gamma_{I} Z_{t}$. ${ }_{1}+\ldots+\Gamma_{\mathrm{k}-1} \Delta Z_{t-k-1}+\prod Z_{t-1}+\mu+\Psi D_{t}+\varepsilon_{t}$. Donde $Z_{\mathrm{t}}$ es un vector de variables no estacionarias, en este caso el PIB, AT y TCR, $\mu$ es el término constante, $D_{t}$ es un vector de variables no estacionarias, $K$ corresponde a la longitud del rezago, $\mathrm{t}=1, \ldots, \mathrm{T}, \varepsilon_{t}$ es el término error. Si los datos son integrados de de primer orden $I(1)$, entonces la matriz $\Pi$ tendrá un rango reducido $r$, es decir, si $r(\Pi)<\mathrm{n}$, entonces existe una reparametrización de $\Pi$ tal que $\Pi=\alpha \beta^{\prime}$ donde $\alpha$ y $\beta$ son matrices de $(n x r)$, donde $\beta^{\prime} Z$ es la matriz de cointegración, es decir, corresponde a las relaciones de cointegración de largo plazo y $\alpha$ representa el parámetro de corrección de error, el cual pude ser interpretado como parámetro de velocidad de ajuste.

Para contrastar el número de vectores de cointegración (o el rango de P) Johansen y Joselius (1990) y Johansen (1988) proponen dos pruebas estadísticas en el modelo de vectores autoregresivos (VAR), la prueba de la Traza $(T r)$, y la prueba de máximo valor propio $(L-M a x)$. En esta prueba la hipótesis nula es la existencia de $r$ vectores de cointegración, contra la alternativa de $r+1$ vectores de cointegración. Así la hipótesis nula $r=0$ es probada contra la alternativa de que $r=1$, y así sucesivamente.

\section{Resultados}

Es necesario precisar algunos hechos relevantes para el estudio: en primer lugar, la serie temporal del PIB inicia en 1995, fecha en que la economía mexicana sufrió un colapso; en segundo lugar se aprecia una contracción de 
manera simultánea de ambas variables en los últimos ańos del periodo de estudio, como consecuencia de la crisis mundial registrada en 2008 originada en Estados Unidos de norteamérica. Cabe mencionar que el quiebre registrado en AT se asocia al impacto de la crisis mundial sobre los turistas norteamericanos, ya que éstos constituyen el principal origen de los turistas internacionales a este destino. Además, se observa que ambas variables tienden a moverse en forma conjunta en el tiempo, lo cual sugiere que estas variables podrían estar cointegradas.

Las gráfica 3 y 4 proporcionan una idea más clara de que puede existir una relación estable a largo plazo el PIB nacional y la afluencia turística extranjera al cip Los Cabos. En la gráfica se puede apreciar la dinámica del PIB, expresado en millones de pesos, a precios de 2002, así como la evolución en el tiempo de AT. Se observa que ambas variables presentan, aparentemente, un crecimiento sostenido en el periodo en cuestión.

\section{Gráfica 3 y 4}

Evolución histórica del PIB (LPIB) y AT (LTUR)
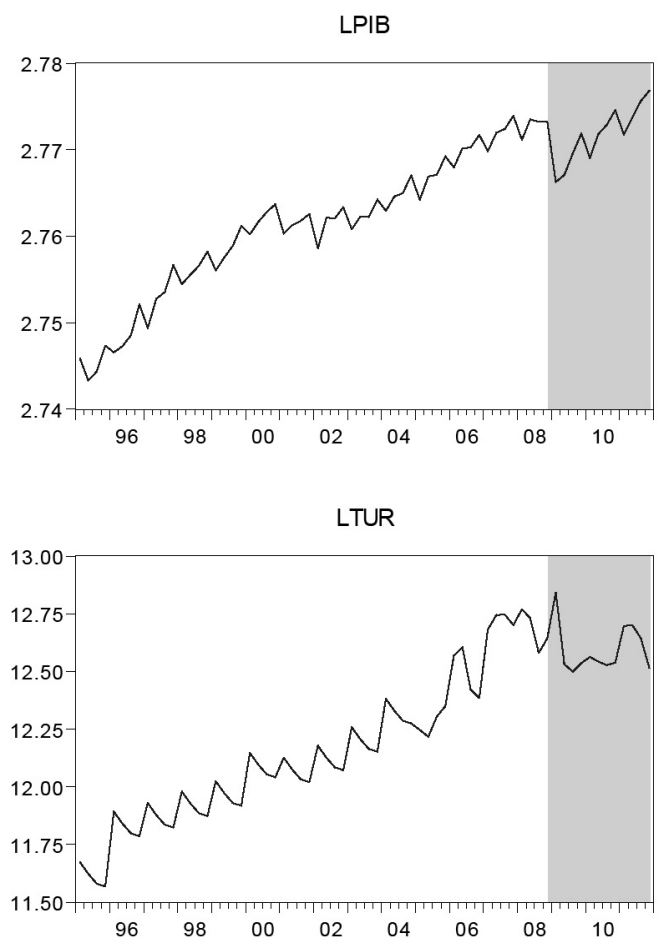

Fuente: elaboración propia. 
Para contrastar lo anterior se propone la siguiente aproximación econométrica en forma logarítmica lineal de la forma $L Y_{t}=\mathrm{c}+\beta_{1} L A T_{t}+\beta_{2} L T C R_{t}+\varepsilon_{t}$. Donde, LY es el logaritmo natural del PIB a precios constantes (base 2002), LAT corresponde al logaritmo natural de AT y LTRC representa el logaritmo natural tipo de cambio real bilateral (TCR) y $\varepsilon$ el termino error.

Debido a que en la literatura empírica macroeconómica las variables del PIB y el TCR se caracterizan por ser no estacionarias se realizaron pruebas para contrastar la estacionareidad de las series, con el fin de evitar problemas de regresión espuria. En este sentido, se aplicó el procedimiento desarrollado por Engle y Granger (1987) mediante prueba de Dickey y Fuller aumentada (1988) y Kwiatkowski et al. (1992). De lo general a lo particular, los rezagos fueron determinados por los criterios de información básico (Akaike y de Schwarz). Los resultados se muestran en la tabla 1.

Tabla 1

Pruebas de raíces unitarias

\begin{tabular}{lcccc}
\hline \multicolumn{1}{c}{ Variables } & ADF & ${ }^{*}$ MacKinnon & KPSS & ${ }^{*}$ KPSS \\
\hline $\ln Y$ & -2.87 & 3.48 & 0.1710 & 0.146 \\
$\mathrm{D} \ln Y$ & -8.92 & 3.48 & 0.1019 & 0.146 \\
$\ln \mathrm{AT}$ & -2.96 & 3.48 & 0.2235 & 0.146 \\
$\mathrm{D} \ln \mathrm{AT}$ & -7.35 & 3.48 & 0.0651 & 0.146 \\
$\operatorname{lnTCR}$ & -1.72 & 3.48 & 1.0315 & 0.466 \\
DlnTCR & -4.36 & 3.48 & 0.20821 & 0.466 \\
*Valores críticos al 5\% & & & & \\
\hline
\end{tabular}

Fuente: elaboración propia.

Los resultados de las pruebas de raíces unitarias indican que no es posible rechazar la hipótesis nula de no estacionareidad a 5\% de significancia. Sin embargo, tomando primeras diferencias, la hipótesis nula de no estacionaridad es rechazada en todas las variables. Por lo tanto, se concluye que las tres variables son integradas de orden uno $I$ (1). Esto es consistente con Nelson y Plosser (1982), quienes encontraron que la mayoría de las variables macroeconómicas son no estacionarias en niveles, pero estacionarias en primeras diferencias. Sabiendo que las series son $I$ (1), se estimó la ecuación $L Y_{\mathrm{t}}=\mathrm{c}+\beta_{l} L A T_{t}+\beta_{2} L T C R_{t}+\varepsilon_{t}$ para contrastar si las variables están cointegradas (tabla 2 ).

Los resultados de la ecuación cointegrante resultaron con los signos esperados indicando que una expansión en el desarrollo turístico tiene un efecto positivo sobre el PIB, mientras que una depreciación de TCR se asociaría con una contracción de la actividad económica nacional. Cabe 
mencionar que los coeficientes de estas variables resultaron significativos a $1 \%$ de significancia.

No obstante, como las series son integradas del mismo orden, se desarrollaron contrastes a los residuales para detectar si los residuales contienen tendencia estocástica o, por lo contrario, son una secuencia estacionaria y determinar si los tres procesos son cointegrados.

El procedimiento del contraste consistió en estimar un modelo de regresión de los residuales rezagados un periodo sobre los residuos en primeras diferencias. La hipótesis nula es la ausencia de cointegración frente a la alternativa de que los procesos están cointegrados y existe al menos un espacio de cointegración para el modelo especificado (tabla 3).

Tabla 2

\section{Ecuación cointegrante: variable dependiente $\operatorname{LnY}$}

\begin{tabular}{lcccc}
\hline \multicolumn{1}{c}{ Variable } & Coeficiente & Error estándar & t-estadistico & Prob. \\
\hline Constante & 2.553 & 0.019 & 129.0161 & 0.00 \\
LTCR & -0.019 & 0.003 & -5.163559 & 0.00 \\
LAT & 0.020 & 0.001 & 15.88622 & 0.00 \\
R-cuadrada & 0.879 & Criterio de información de Akaike & -8.71 \\
R-cuadrada ajustada & 0.875 & Criterio de información de Schwarz & -8.61 \\
S.E. de la regresión & 0.003 & Estadístico-F & 235.09 & \\
Suma de residuos al & 0.001 & Prob. & 0.00 & \\
cuadrado & & & 68 & \\
Durbin-Watson & 1.663 & Observaciones & & \\
\hline
\end{tabular}

Fuente: elaboración propia.

Tabla 3

Prueba de cointegración, variable dependiente: D (residuos)

\begin{tabular}{lrlcr}
\hline \multicolumn{1}{c}{ Variable } & Coeficiente & Error estándar & Estadistico $t$ & Prob. \\
\hline Constante & $-6.80 \mathrm{E}-06$ & 0.0004 & -0.0184 & 0.98 \\
Residuos (-1) & -0.887 & 0.1328 & -6.6784 & 0.00 \\
R-cuadrada & 0.407 & Criterio de información de Akaike & -8.739 \\
R-cuadrada ajustada & 0.398 & $\begin{array}{l}\text { Criterio de información de } \\
\text { Schwarz }\end{array}$ & -8.673 \\
$\begin{array}{l}\text { S.E. de la regresión } \\
\text { Suma de residuos al } \\
\text { cuadrado }\end{array}$ & 0.003 & Estadístico-F & 44.60 \\
Durbin-Watson & 0.001 & Prob. & 0.00 \\
\hline
\end{tabular}

Fuente: elaboración propia. 
Como se aprecia en la tabla 3, la prueba de los residuos exhibió un estadístico $t$ que rechaza la hipótesis nula de no cointegración, lo que sugiere la existencia de una relación estable a largo plazo, así como la presencia de un espacio de cointegración en el modelo especificado. En este sentido, el Teorema de Representación de Granger (TGR) indica que si existe cointegración también existirá un Mecanismo de Corrección de Error (MCE) para representar el Proceso Generador de los Datos (PGD).

Para incorporar la representación de largo plazo en una de corto plazo que corrija el error que le es consustancial, se estimó el modelo en primeras diferencias y, de acuerdo a Hendry (1995) y Jhonston y Dinardo (1997), se definió el modelo como un procedimiento para estimar resultados de rezagos diferentes en las variables explicatorias, obteniendo la selección óptima al minimizar los criterios de de Schwarz y Akaike. De acuerdo al TGR se estimó una ecuación que incorpora el MCE, $\Delta L Y_{t}=c+\alpha_{1} \Delta L A T_{t}+\alpha_{2} \Delta L T C R_{t}+\alpha_{3}\left(L Y_{t-1}-c-\beta_{l} L A T_{t-1}-\beta_{2} L T C R_{t-1}\right)-\varepsilon_{t}$.

Los resultados del MCE se muestran en la tabla 4 , y se incorporan las pruebas de diagnóstico para detectar presencia de correlación serial, heteroscedasticidad, y normalidad de los residuos. Las cuales se deduce que los errores están normalmente distribuidos y no presentan problemas de autocorrelación, ni heteroscedasticidad.

Los resultados del MCE muestran que los coeficientes de las variables en primeras diferencias tienen signos iguales a los obtenidos en la regresión cointegrante y estadísticamente significativos a 5 por ciento. De esta manera el MCE muestra consistencia de los coeficientes y efecto sobre el PIB, es decir, el incremento en AT está asociado al crecimiento económico nacional, aun cuando la magnitud sea relativamente pequeńa. En cuanto al TCR, los resultados indican que una depreciación de esta variable tiene un efecto negativo sobre la actividad económica nacional. El coeficiente que representa el mecanismo de corrección de error, resultó estadísticamente significativo a $1 \%$ y su magnitud es menor en términos absolutos a la unidad, lo que indica que cualquier desequilibrio de la relación de largo plazo derivado del corto plazo se corrige en 38\% trimestral, lo cual valida que la relación entre AT con el PIB es estable a largo plazo.

En cuanto al TCR se confirman los resultados encontrados por la literatura empírica para México de que se encuentra asociada a contracciones de la actividad económica. Adicionalmente se incorporó una variable dicotómica que capta el efecto de negativo de la última crisis financiera para representar el efecto de la crisis financiera durante el cuarto trimestre de 2008 y el primero de 2009. Cabe mencionar que las pruebas de diagnostico del modelo econométrico presentadas en la tabla 4, revelan solidez desde el punto de vista estadístico. 
Tabla 4

Modelo de corrección de error

Variable dependiente: PIB

\begin{tabular}{|c|c|c|c|c|}
\hline Variable & Coeficiente & Error estándar & Estadistico $t$ & Prob. \\
\hline Constante & 0.0005 & 0.000 & 3.122 & 0.00 \\
\hline $\mathrm{D}($ LPIB $(-3))$ & -0.3537 & 0.092 & -3.853 & 0.00 \\
\hline $\mathrm{D}(\operatorname{LTCR}(-1))$ & -0.0095 & 0.004 & -2.262 & 0.03 \\
\hline $\mathrm{D}(\mathrm{LAT}(-3))$ & 0.0043 & 0.002 & 2.439 & 0.02 \\
\hline $\operatorname{RE}(-1)$ & -0.3847 & 0.059 & -6.544 & 0.00 \\
\hline dummy & -0.0026 & 0.001 & -2.664 & 0.01 \\
\hline R-cuadrada & 0.65 & Akaike AIC & & -10.38 \\
\hline R-cuadrada ajustada & 0.62 & Schwarz SC & & -10.18 \\
\hline S.E. de la regresión & 0.0013 & Estadístico F & & 21.97055 \\
\hline $\begin{array}{l}\text { Suma de residuos al } \\
\text { cuadrado }\end{array}$ & $9.57 \mathrm{E}-05$ & Prob. & & 0.00 \\
\hline Durbin-Watson & 1.68 & Observaciones & & 64 \\
\hline $\begin{array}{l}\text { Breusch-Godfrey: } \\
2 \text { rezagos } 2.49(\mathrm{p}=0.28)\end{array}$ & & \multicolumn{3}{|c|}{ White: sin ordenada $7.01 \quad(p=0.63)$} \\
\hline $\begin{array}{l}\text { Breusch-Godfrey: } \\
3 \text { rezagos } 5.37(\mathrm{p}=0.14)\end{array}$ & & \multicolumn{3}{|c|}{ White: conn ordenada $7.01(\mathrm{p}=0.63)$} \\
\hline $\begin{array}{l}\text { Breusch-Godfrey: } \\
4 \text { rezagos } 6.71(p=0.15)\end{array}$ & & \multicolumn{3}{|c|}{ Jarque-Bera: $4.73(\mathrm{p}=0.10)$} \\
\hline
\end{tabular}

Fuente: elaboración propia.

Sin embargo, el modelo de cointegración desarrollado por Engle y Granger, presenta el inconveniente de que en modelos de más de dos variables, como el de este trabajo, sólo es posible estimar un vector de cointegración, lo cual puede llevar a pérdida de información relevante.

Con el fin de evaluar si en efecto existe un único vector cointegrador, se aplica el enfoque multivariante de Johansen y Joselius (1990) y Johansen (1998), esto con el fin de contrastar el número de vectores de cointegración (o el rango de $\mathrm{P}$ ); los resultados y las respectivas pruebas de contraste se muestran en la tabla 5. 


\section{Tabla 5}

\section{Prueba de cointegración de Johansen o de rango no restricta}

\begin{tabular}{lcccc}
\hline \multicolumn{5}{c}{ Supuesto: No hay tendencia deterministica } \\
Series: $P I B, A T$, TCR \\
$\begin{array}{c}\text { Ho: rango }= \\
p H_{0}: \text { rango }=p\end{array}$ & Valor propio & Estadistico & Valor critico & Prob. \\
\hline Tr & & & & \\
$\mathrm{P}=0^{*}$ & 0.40 & 43.8 & 29.8 & 0.00 \\
$\mathrm{P} \leq 1$ & 0.11 & 9.92 & 15.49 & 0.29 \\
$\mathrm{P} \leq 2$ & 0.03 & 1.93 & 3.84 & 0.16 \\
L-Max & & & & \\
$\mathrm{P}=0^{*}$ & 0.40 & 33.88 & 21.13 & 0.00 \\
$\mathrm{P} \leq 1$ & 0.11 & 7.99 & 14.26 & 0.38 \\
$\mathrm{P} \leq 2$ & 0.03 & 1.93 & 3.84 & 0.16 \\
\hline
\end{tabular}

* Rechazo de la hipótesis nula a 5\% de significancia.

P: número de vectores de cointegración.

Fuente: elaboración propia.

De acuerdo con la prueba de la traza $(T r)$ rechaza la hipótesis nula (Ho: $\mathrm{P}=0$ ) de no cointegración en favor de una relación de cointegración al nivel del $5 \%(43.80>29.80)$. Igualmente la prueba $L-M a x$ indica la existencia de una sola ecuación de cointegración tanto al 5\% (33.88>21.13). De los resultados de $\operatorname{Tr}$ y $L$-Max, se concluye que existe un solo vector o relación de cointegración, lo que confirma la relación de largo plazo entre las variables estudiadas y confirma los resultados del anterior modelo de MCE.

\section{Tabla 6}

\section{Prueba de exogeneidad débil de AT y vector de cointegración}

Restricciones de cointegración: $A(2,1)=0, B(1,1)=1$

Prueba LR para las restricciones (rango $=1$ )

$\chi 2(1): 17.50$

Probabilidad: 0.00

Ecuación de cointegración después de prueba de exogeneidad

\begin{tabular}{lcccc} 
& PIB & TCR & AT & Constante \\
Coeficiente & 1 & 0.0644 & -0.0209 & -2.6247 \\
Error estándar & & -0.0129 & -0.0041 & \\
Estadístico-t & & {$[5.0034]$} & {$[-5.0594]$} & \\
\hline
\end{tabular}

Fuente: elaboración propia. 
No obstante, debido a que la relación de cointegración no aporta información sobre la dirección de causalidad entre las variables, ni cuál o cuáles variables pueden considerarse exógenas, es necesario contrastar la condición de exogeneidad débil de AT, añadiendo una restricción a la matriz $\alpha$ del Modelo de Corrección del Error en Forma Vectorial (VECM). En la tabla 6, se puede apreciar la relación de cointegración después de probar la exogeneidad débil.

De acuerdo a la prueba LR con una restricción, la variable AT puede ser considerada como débilmente exógena. Al probar la exogeneidad débil de AT es posible usar las ecuaciones estimadas sin necesidad de modelar la variable en el modelo. Para normalizar este vector de cointegración se multiplican los parámetros estimados por -1 y se reordenan los términos de tal manera que el vector se interprete como una función de producto. En este sentido, la ecuación de cointegración por el método de Johansen se define de la siguiente forma:

$$
\mathrm{PIB}_{\mathrm{t}}=2.62+0.02 \mathrm{AT}_{\mathrm{t}}-0.06 \mathrm{TCR}_{\mathrm{t}}
$$

Esta ecuación revela que la elasticidad del PIB con respecto a la AT es 0.02. Esto significa que, ceteris paribus, un incremento en AT en Los Cabos de $1 \%$ provoca (a largo plazo) un aumento de $0.02 \%$ del piB. Mientras que, ceteris paribus, un aumento de $1 \%$ en el TCR provoca una disminución de 0.06 en el pib. Los signos de los parámetros de las variables coinciden con los de la ecuación MCE.

El análisis de cointegración sólo indica asociación a largo plazo pero no relación causal. Granger (1998) propone sea aplicada al VECM una prueba para estudiar la causalidad, la cual consiste en determinar si una variable endógena puede ser tratada como exógena. Busca determinar estadísticamente si el pasado de una variable contiene información que preceda al comportamiento de otra variable, por tanto contribuye a su explicación. La hipótesis nula es que AT no explica (o no causa) el PIB, en términos formales, Ho: la afluencia extranjera a Los Cabos (AT) no causa el PIв (tabla 7).

Los resultados muestran que Ho se rechaza y se interpretan de forma que AT y TCR determinan el PIB, mientras que el PIB determina la AT, lo cual sugiere que la relación de causalidad va en ambos sentidos, es decir, es bidireccional. Estos resultados brindan evidencia de que la relación entre AT y PIB, no es una relación espuria, sino que ambas guardan una relación de equilibrio a largo plazo. Estos resultados tienden a favorecer la TLGH para Los Cabos durante el periodo de estudio, además es consistente con la literatura empírica presentada anteriormente, por lo que se puede inferir una relación bidireccional entre ambas variables. 


\section{Tabla 7}

\section{Prueba de causalidad de Granger}

\begin{tabular}{lrr}
\hline \multicolumn{1}{c}{ Variable dependiente: } & \multicolumn{1}{c}{$\chi^{2}$} & \multicolumn{1}{c}{ Probabilidad } \\
\hline TCR no causa a PIB & $43.23595^{* *}$ & 0.0000 \\
AT no causa a PIB & $46.3087^{* *}$ & 0.0000 \\
PIB no causa a TCR & 0.123821 & 0.9800 \\
AT no causa a TCR & 2.507731 & 0.4700 \\
PIB no causa a AT & $18.62537^{* *}$ & 0.0000 \\
TCR no causa a AT & 1.825353 & 0.6100 \\
\hline
\end{tabular}

Nota: *, ** Indican el rechazo de la hipótesis nula al 5\% y al 1\%, respectivamente.

Fuente: elaboración propia.

Los coeficientes del MCE y del VECM tienen los signos esperados, aunque deben entenderse como una forma reducida y por tanto sin interpretación directa. Para representar la relación dinámica a largo plazo se estimó un modelo de Vectores Autoregresivos con Corrección de Error, caracterizado por considerar a todas las variables como endógenas e incorporarlas en un sistema donde son función de los valores rezagados de esas variables.

La ecuación (3) revela que la relación de las variables en el sistema presenta signos iguales a los obtenidos en los modelos de regresión antes estimados, donde AT está positivamente relacionado con el crecimiento económico de largo plazo, y TCR es consistente con los reportados por la literatura del crecimiento económico en México, donde se muestra empíricamente que existe una asociación de la depreciación del TCR y una contracción de la actividad económica.

$$
\begin{aligned}
\Delta P I B_{t}= & 0.10\left[P I B_{t-1}+2.62+0.02 A T_{t}-0.06 T C R_{t}\right](3.68) \\
& -0.68 \Delta P I B_{t-1}(6.95)-0.32 \Delta P I B_{t-2}(-3.24)-0.49 \Delta P I B_{t-3}(-5.83) \\
& -0.01 \Delta T C R_{t-1}(-4.37)-0.014 \Delta T C R_{t-2}(-4.42)-0.006 \Delta T C R_{t-3}(-1.78) \\
& +0.003 \mathrm{AT}_{t-1}(2.06)+0.007 \Delta A T_{t-2}(4.56)+0.006 A T_{t-3}(4.34)
\end{aligned}
$$

$$
\begin{aligned}
\Delta A T_{t}= & 22.65 \Delta P I B_{t-1}(2.87)+12.75 \Delta P I B_{t-2}(1.58)+18.42 \Delta P I B_{t-3}(2.72) \\
& +0.17 \Delta T C R_{t-1}(0.61)-0.18 \Delta T C R_{t-2}(-0.70)+0.11 \Delta T C R_{t-3}(0.41) \\
& -0.16 A T_{\mathrm{t}-1}(-1.13)-0.51 \Delta A T_{t-2}(-4.16)-0.19 A T_{t-3}(-1.54) \\
\Delta T C R_{t}= & 5.40 \Delta P I B_{t-1}(1.81)+1.67 \Delta P I B_{t-2}(0.54)-1.15 \Delta P I B_{t-3}(-0.05) \\
& +0.05 \Delta T C R_{t-1}(0.52)-0.04 \Delta T C R_{t-2}(-0.48)+0.08 \Delta T C R_{t-3}(0.84) \\
& +0.08 A T_{\mathrm{t}-1}(0.14)+0.01 \Delta A T_{t-2}(-0.27)+0.05 A T_{t-3}(1.10)
\end{aligned}
$$


Para contrastar la robustez del vecm se realizaron las pruebas de diagnóstico de normalidad de Doornick y Hansen (2008) y Lütkepohl (1993), y la prueba LM de Breusch-Godfrey para probar autocorrelación en los residuos (anexos 4 y 5). Del anexo 4 se deduce que los errores se distribuyen de manera normal, individual y conjuntamente, mientras que en el anexo 5 la prueba LM indica que los errores no tienen problemas de autocorrelación.

Adicionalmente para contrastar la estabilidad del modelo y la causalidad entre las variables, se realizo un análisis de impulso respuesta. Como se aprecia en los resultados de la función impulso respuesta (Anexo 6) del PIB a un impacto (shock) en AT es positiva, sin embargo después del cuarto trimestre empieza a desvanecerse hasta desaparecer asintóticamente. Por otra parte se observa que el PIB reacciona al impulso del TCR, pero al tercer trimestre este efecto tiende a desvanecerse. Estos resultados permiten confirmar los resultados obtenidos con el modelo de dos etapas de Engle y Granger, teniendo dos implicaciones importantes, primera, permiten confirmar el cumplimiento de la TLGH en el cip de Los Cabos y; segunda, da pauta para evaluar de manera positiva la política sectorial del turismo en este destino turístico.

\section{Conclusión}

Para hacer que este Cip despegará las acciones de política de desarrollo turístico se centraron, en sus inicios, en la creación de infraestructura, mejora de la conectividad (aérea y de telecomunicaciones), en la construcción de hoteles, servicios de apoyo y otras instalaciones turísticas desde sus inicios hasta el final del periodo de estudio. Por otra parte, durante las últimas dos décadas del siglo pasado se dieron fuertes inversiones en infraestructura hotelera, principalmente por parte de la iniciativa privada, así como por FONATUR en la zona del cip Los Cabos; además de inversiones complementarias realizadas por el Gobierno del Estado de Baja California Sur. Todas ellas encaminadas a la promoción, consolidación y posicionamiento del cip Los Cabos como destino de sol y playa internacional. En la primera década del siglo xxi Los Cabos avanza en franco auge, derivado de las acciones anteriores de política de desarrollo turístico; hasta fines de la misma, donde su dinámica se ve frenada por las consecuencias de la crisis financiera de 2008 y el brote del virus AH1N1; sin embargo, los resultados de este estudio muestran que Los Cabos se está recuperando de este shock.

Las series temporales muestran gráficamente el comportamiento de la dinámica del crecimiento económico nacional y del desarrollo del cIP Los Cabos, mediante los arribos de turistas internacionales (АT). Los 
resultados de las estimaciones econométricas mediante el método de Engle y Granger y por el método de Johansen y Joselius muestran que la hipótesis planteada para el primer objetivo es correcta, es decir, se corrobora la existencia de una relación estable de largo plazo entre el PIB de México y AT para el periodo en cuestión. Además, se observa que las técnicas utilizadas son estadísticamente aceptables para corroborarlo, ya que los resultados de ambos modelos satisfacen la relación entre el PIв у Ат al $95 \%$ de confianza.

El segundo objetivo particular no se puede comprobar a cabalidad, ya que los métodos de cointegración manifiestan una relación bidireccional entre el crecimiento económico nacional y la afluencia turística internacional a Los Cabos. Es decir, se corrobora la TLGH al haber encontrado una relación bidireccional entre el PIB y AT, pero no se encuentra una relación causal entre ellas. Lo cual conlleva a que este estudio se sume a los demás que no han podido establecer una relación unidireccional entre estas variables. Si bien la TLGH se ha comprobado para México a nivel macroeconómico por Brida et al. (2008a), ésta no había tratado de ser validada a nivel local o regional, y particularmente en un cIP tan dinámico como Los Cabos.

En lo concerniente al tercer objetivo propuesto en este estudio, se puede corroborar la existencia de una relación positiva entre AT y el PIB. La magnitud de tal relación es relativamente pequeña (alrededor de 0.004\%), considerando la escala del desarrollo turístico en el CIP Los Cabos, con la actividad económica nacional. Los resultados dejan ver que el cip Los Cabos contribuye positivamente en el crecimiento económico nacional de largo plazo.

El que su magnitud sea pequeña resalta que las estimaciones son consistentes y coherentes. Un valor más elevado podría llevar a inferir un crecimiento mayor que otros sectores más importantes en el crecimiento económico de México. Teniendo en cuenta que la wTTc indica que el turismo internacional contribuye con 5.9\% del рів nacional, comparando esta cifra con $0.004 \%$ que el cip Los Cabos aporta a la economía mexicana, es posible afirmar que el estudio permite evaluar, en el largo plazo, la política de desarrollo turístico planteada por México mediante la creación de los cip, entre ellos el de Los Cabos.

Finalmente, en la función impulso-respuesta se deben destacar dos aspectos, uno, un shock en AT se corrige en 38\% de forma trimestral (LTUR-PIB). Esta recuperación se estará viendo en aproximadamente cinco años y dos meses a partir del momento que ocurrió el shock. Dos, una depreciación del TCR tiene un efecto negativo sobre la actividad económica nacional, este efecto se desvanece en aproximadamente tres años (LPIB-LTCR). Las estimaciones hechas en el estudio ponen en relevancia 
que se ha dado una recuperación de la caída de AT causada por la crisis económica mundial y los efectos del AH1N1.

\section{Fuentes consultadas}

Akinboade, Oludele A. y Lydia Braimoh (2010), "International tourism and economic development in South Africa: a Granger causality test", International Journal of Tourism Research, 12 (2), Wiley, Hoboken, pp. 146-163, <doi: 10.1002/jtr.743>.

Arslanturk, Yalcin, Mehmet Barcilar y Zeynel Abidin Ozdemir (2011), "Time-varying linkages between tourism receipts and economic growth in a small open economy", Economic Modelling, 28 (1-2), Elsevier, Amsterdan, pp. 664-671.

Balaguer, Jacint y Manuel Cantavella-Jordá (2002), “Tourism as a longrun economic growth factor: the Spanish case", Applied Economics, 34 (7), Taylor and Francis, Londres, pp. 877-884.

Belloumi, Mounir (2010), "The relationship between tourism receipts, real effective exchange rate and economic growth in Tunisia", International Journal of Tourism Research, 12 (5), Wiley, Hoboken, pp. 550-560.

Billfish Foundation (2008), "Contribución económica de la pesca deportiva a la economía de Los Cabos", Sportfishing Socioeconomic Studies, <http://www.billfish.org/research/socioeconomics/>, 29 de octubre de 2013.

Brida, Juan Gabriel, Pablo Daniel Monterubbianesi y Sandra ZapataAguirre (2011), "Impactos del turismo sobre el crecimiento económico y el desarrollo. El caso de los principales destinos turísticos de Colombia”, PASOS Revista de turismo y patrimonio cultural, 9 (2), Universidad de La Laguna, Tenerife, pp. 291-303.

Brida, Juan Gabriel, Andrea Barquet y Wiston Adrian Risso (2009), "Causality between economic growth and tourism expansion: empirical evidence from Trentino-Alto Adige", Tourismos: an international multidisciplinary journal of tourism, 5 (2), University of the Aegean, Aegean, pp. 87-98. 
Brida, Juan Gabriel, Juan S. Pereyra, Wiston Adrian Risso, María Jesús Such-Devesa y Sandra Zapata-Aguirre (2009), "The tourism growth hypothesis: empirical evidence from Colombia", Tourismos: an international multidisciplinary journal of tourism, 4 (2), University of the Aegean, Aegean, pp. 13-27.

Brida, Juan Gabriel, Bibiana Lanzilotta y Wiston Adrian Risso (2008a), "Turismo y crecimiento económico: el caso de Uruguay", PASOS Revista de turismo y patrimonio cultural, 6 (3), Universidad de La Laguna, Tenerife, pp. 481-492 pp.

Brida, Juan Gabriel, Edgar Javier Sánchez-Carrrera y Wiston Adrian Risso (2008b), "Tourism's impact on long-run mexican economic growth", Economics Bulletin, 3 (21), Social Science Research Network, pp 1-8.

Castillo-Ponce, Amadeo Ramón y Rogelio Varela-Llamas (2010), Econometría práctica: fundamentos de series de tiempo, Universidad Autónoma de Baja California, Baja California.

Centro de Estudios de Finanzas Públicas del H. Congreso de la Unión (2010), Publicaciones macroeconómicas, estadísticas, <http://www. cefp.gob.mx/Pub_Macro_Estadisticas.htm>, febrero de 2010.

Chen, Ching Fu y Song Zan Chiou-Wei (2008), “The tourism growth hypothesis: empirical evidence from Colombia”, Tourism Management, 30, Elsevier, Amsterdam, pp. 812-818.

Cortés-Jiménez, Isabel y Manuela Pulina (2006) "A further step into the ELGH and TLGH for Spain and Italy", note di lavoro, Fondazione Eni Enrico Mattei, <http://www.feem.it/NR/rdonlyres/ C6F679FC-64E0-4CA5-AB1E-943B04B9A241/2375/11808. pdf>, 1 de noviembre de 2012.

Croes, Robertico y Manuel Vanegas (2008), "Cointegration and causality between tourism and poverty reduction", Journal of Travel Research, 47 (1), Sage Publications, Reino Unido, pp. 94-103.

De la Cruz-Gallegos José Luis, Carlos Canfield-Rivera y J. Núñez-Mora (2010), "Drivers of economic growth: the case for tourism in Mexico", Revista Brasileira de Economía Empresarial, 10 (2), Fundação Getúlio Vargas, Rio de Janeiro, pp. 38-53. 
Dickey, David y Wayne Fuller (1988), "Likelihood ratio statistics for autoregressive time series with unit root”, Econométrica, 49 (4), Wiley, Hoboken, pp. 1057-1072.

Ditton, Robert, Sheperd Grimes y Leslie Filkenstein (1996), “A social and economic study of the recreational billfish fishery in the Southern Baja Area of Mexico", Reporte preparado para Billfish Foundation, Texas A \& M University.

Doornik, J. A. y H. Hansen (2008), "An omnibus test for univariate and multivariate normality", Oxford Bulletin of Economics and Statistics, 70 (1), Wiley, Oxford, pp. 927-939.

Dritsakis, Nikolaos (2004), “Tourism as a long-run economic growth factor: an empirical investigation for Greece using a causality analysis", Tourism Economics, 10 (3), IP Publishing LTD, Londres, pp. 305-316.

Engle, Robert y Clive Granger (1987), "Co-integration and error correction: representation, estimation, and testing", Econometrica, 55 (2), Wiley, Hoboken, pp. 251-276.

Galindo, Luis Miguel y Carlos Guerrero (1997), "Factores determinantes de la balanza comercial en México 1980-1995", Revista de Comercio Exterior, 47 (10), Bancomext, México, pp. 789-794.

Galindo, Luis Miguel y Carlos Guerrero (2001), "Los efectos de la recesión estadounidense y del tipo de cambio sobre el crecimiento económico de México en 2001", Momento económico, núm. 116, Universidad Nacional Autónoma de México, México, pp. 2-9.

Garcés-Díaz, Daniel (2006), "La relación de largo plazo del piB mexicano y sus componentes con la actividad económica de Estados Unidos y el tipo de cambio real", Economía mexicana, XV (1), Centro de Investigación y Docencia Económica, México, pp. 5-30.

Gardella, Rodrigo y Eva Aguayo (2002), "Impacto económico del turismo en el Mercosur y Chile (1990-2000)", Estudios económicos de desarrollo regional, 2 (1), Econpapers, Madrid, pp. 27-49.

Gobierno del Estado de Baja California Sur (2009), Quinto informe de gobierno: Narciso Agúndez Montaño, documento gráfico, La Paz. 
Granger, Clive (1988), "Some recent developments in a concept of causality”, Journal of Econometrics, vol. 39, Elsevier, 199-211 pp.

Gunduz, Lokman y Abdulnasser Hatemi-J. (2005), "Is the tourism-led growth hypothesis valid for Turkey?", Applied Economics Letters, 12 (8), Taylor and Francis, Londres, 499-504 pp.

Hendry, David (1995), Dynamics Econometrics. Advanced texts in econometrics, Oxford University Press, Oxford.

Husein, Jamal y Sahit Murat Kara (2011), "Research note: re-examining the tourism-led growth hypothesis for Turkey", Tourism Economics, 17 (4), IP Publishing LTD, Londres, pp. 917-924.

INEGI (Instituto Nacional de Estadística, Geografía e Informática) (2012a), Encuesta Nacional de Ocupación y Empleo, INEGI, <http://www. inegi.org.mx/lib/olap/consulta/general_ver4/MDXQueryDatos. asp?\#Regreso\&c=10823>, febrero de 2012.

INEGI (Instituto Nacional de Estadística, Geografía e Informática) (2012b), Censos económicos, INEGI, <http://www.inegi.org.mx/est/contenidos/proyectos/ce/>, febrero de 2012.

INEGI (Instituto Nacional de Estadística Geografía e Informática) (2010), Banco de Información Económica, México, <http://www.inegi. org. $\mathrm{mx} /$ sistemas/bie/>, enero de 2010 .

Jhonston, John y John Dinardo (1997), Econometric methods, McGrawHill, Nueva York.

Johansen, Søren y Katarina Joselius (1990), "Maximum likelihood estimation and inference on cointegration-with applications to the demand for money", Oxford Bulletin of Economics and Statistics, 52 (2), Wiley, Hoboken, pp. 169-210.

Johansen, Søren (1988), "Statistical analysis of cointegration vectors", Journal of Economic Dynamics and Control, 12 (2-3), Elsevier, Amsterdam, pp. 231-254.

Kadir, Norsiah y Kamaruzaman Jusoff (2010), "The cointegration and causality tests for tourism and trade in Malaysia", International 
Journal of Economics and Finance, 2 (1), The Canadian Center of Science and Education, Toronto, pp. 138-143.

Kamin, Steve B. y John Rogers (1997), "Output and the real exchange rate in developing countries: an aplication to Mexico", International Finance Discussion paper, 61 (1), Board of Governors of the Federal Reserve System, Washington.

Kaplan, Muhittin y Celik, Tunkai (2008), "The impact of tourism on Economic Performance: the case of Turkey", The International Journal of Applied Economics and Finance, 2 (1), Asian Networks for Science Information, pp. 13-18, <doi: 10.3923/ijaef.2008. $13.18>$.

Katircioglu, Salih Turan (2010), "Research note: testing the tourism-led growth hypothesis for Singapore - an empirical investigation from bounds test to cointergration and granger causality tests", Tourism Economics 16 (4), IP Publishing Ltd, Londres, pp. 1095-1101.

Katircioglu, Salih Turan (2009a), "Tourism, trade and growth: the case of Cyprus", Applied Economics, 41(21), Universidad del CEMA, Buenos Aires, pp. 2471-2750.

Katircioglu, Salih Turan (2009b), "Testing the tourism-led growth hypothesis: the case of Malta”, Acta Oeconomica, 59 (3), Akadémiai Kiadó, Budapest, pp. 331-343.

Khan, Habibullah, Rex Toh y Lyndon Chua (2005), "Tourism and trade: cointegration and granger causality tests", Journal of Travel Research, 44 (2), Sage Publications, Virginia, pp. 171-176.

Kim, Hyun Jeong, Ming-Hsiang Chen y Soo Cheong Shawn Jang (2006), "Tourism expansion and economic development: the case of Taiwan”, Tourism Management, 27 (5), Elsevier, Amsterdam, pp. 925-933.

Kwiatkowski, Denis, Peter Phillips, Peter Schmidt y Yongcheol Shin (1992), "Testing the null hypothesis of stationarity against the alternative of a unit root: How sure are we that economic time series have a unit root?", Journal of Econometrics, núm. 54 (1-3), Elsevier, Amsterdam, pp. 159-178. 
Lean, Hooi Hooi y Chor Foon Tang (2010), "Is the tourism-led growth hypothesis stable for Malaysia? A note", International Journal of Tourism Research, 12 (4), Wiley, Hoboken, pp. 375-378.

Lee, Chien-Chiang y Chun-Ping Chang (2008), "Tourism development and economic growth: a closer look at panels", Tourism management, 29 (1), Elsevier, Amsterdan, pp. 180-192.

Lee, Chien-Chiang y Mei-Se Chien (2008), "Structural breaks, tourism development, and economic growth: evidence from Taiwan", Mathematics and Computers in Simulation, 77 (4), Elsevier, Amsterdan, pp. 358-368.

López, Julio y Carlos Guerrero (1998), "Crisis externa y competitividad de la economía mexicana”, El Trimestre Económico, LXV (260), Fondo de Cultura Económica, México, pp. 582-598.

Louca, Charalambos (2006), "Income and expenditure in the tourism industry: time series evidence from Cyprus", Tourism Economics, 12 (4), IP Publishing LTD, Londres, pp. 603-617.

Lütkepohl, H. (1993), Introduction to multiple time series analysis, Springer, Berlin.

Magaña-Carrillo, Irma (2009), "La política turística en México desde el modelo de calidad total: un reto de competitividad", Economía, Sociedad y Territorio, IX (30), El Colegio Mexiquense, A. C., Zinacantepec, pp. 515-544.

Molina, Sergio (2008), Política turistica en México, Trillas, México.

Narayan, Paresh Kumar, Seema Narayan, Biman Chand Prasad y Arti Prasad (2007), "Export-led growth hypothesis: evidence from Papua New Guinea and Fiji”, Journal of Economic Studies, 34 (4), Emerald Group Publishing Limited, Bradford, pp. 341-351.

Nelson, Charles y Charles Plosser (1982), “Trends and random walk in macroeconomics", Journal of Monetary Economics, 10 (2), Elsevier, Amsterdam, pp. 139-162.

Nowak, Jean-Jacques, Salih Mondher e Isabel Cortés-Jiménez (2007), "Tourism, capital good imports and economic growth: theory 
and evidence for Spain", Tourism Economics, 13 (4), IP Publishing LTD, Londres, pp. 515-536.

Oh, Chi-Ok (2005), "The contribution of tourism development to economic growth in the Korean economy", Tourism Management, 26 (1), Elsevier, Amsterdam, pp. 39-44.

Ongan, Serdar y Dündar Demiroz (2005), "The contribution of tourism to the Long-Run Turkish economic growth", Ekonomický časopis, (9), Ekonomický ústav SAV, Bratislava, Eslovaquia, pp. 880-894.

Po, Wan-Chen y Bwo-Nung Huang (2008), "Tourism development and economic growth-a nonlinear approach”, Physica A, 387 (22), Elsevier, Amsterdan, pp. 5535-5542.

Quintana-Romero, Luis y Miguel Ángel Mendoza-González (2008), Econometría básica. Modelos y aplicaciones a la economía mexicana, unam-DGapa-Plaza y Valdés, México.

Ramírez-Hernández, J. J. (2006), "Actividad económica del sector turístico mexicano: situación actual, tendencias y cointegración", Aportes. Revista de la Facultad de Economía, XI (31), Benemérita Universidad Autónoma de Puebla, Puebla, pp. 89-106.

Schubert, Stefan Franz, Juan Gabriel Brida y Wiston Adrian Risso (2010), "The impacts of international tourism demand on economic growth of small economies dependent on tourism", Tourism Management, 32 (2), Elsevier, Amsterdan, pp. 377-385.

SIIMT (Sistema Integral de Información de Mercados Turísticos) (2012), Reporte de flujos turísticos a México por Estado, Consejo de Promoción Turística de México, <http://www.siimt.com/en/siimt/ siim_flujos_mensuales>, 15 de noviembre de 2012 .

Subsecretaría de Planeación Turística (2012), Resultados de la actividad hotelera (enero-diciembre 2012), Sectur, <http://www.datatur. beta.sectur.gob.mx/Documentos\%20Publicaciones/sem012013. pdf>, 3 de septiembre de 2012.

Tang, Chor Foon (2011), "Is the tourism-led growth hypothesis valid for Malaysia? a view from disaggregated tourism markets", In- 
ternational Journal of Tourism Research, 13 (1), Wiley, Hoboken, pp. 97-101.

Tang, Chun-Hung y SooCheong Jang (2009), "The tourism-economy causality in the United States: A sub-industry level examination", Tourism Management, 30 (4), Elsevier, Amsterdan, pp. 553-558.

Tello-Contreras, Juan Manuel, Giannina Consuelo Cerda-Martínez y Paloma Pardo-Manzanares (2012), Índice de Competitividad Turística de los Estados Mexicanos-ICTEM 2012, Centro de Investigación y Estudios de Turismo-Tecnológico de Monterrey.

unwTo (United Nations World Tourism Organization) (2014), unwTO World Tourism Barometer, anexo estadístico, vol. 14, UNwTO, Nueva York.

unwto (United Nations World Tourism Organization) (2013), unwTo World Tourism Barometer, 11 (1), Nueva York.

unwto (United Nations World Tourism Organization) (2011), unwTo World Tourism Barometer, actualización provisional, vol. 9, unwto, Nueva York.

WTTC (World Tourism y Travel Council) (2013a), Economic impact 2013, WTTC, Londres.

WTTC (World Tourism y Travel Council) (2013b), Travel y tourism economic impact. México, wTTC, Londres.

Recibido: 6 de julio de 2013. Reenviado: 12 de septiembre de 2014. Aceptado: 30 de septiembre de 2014. 


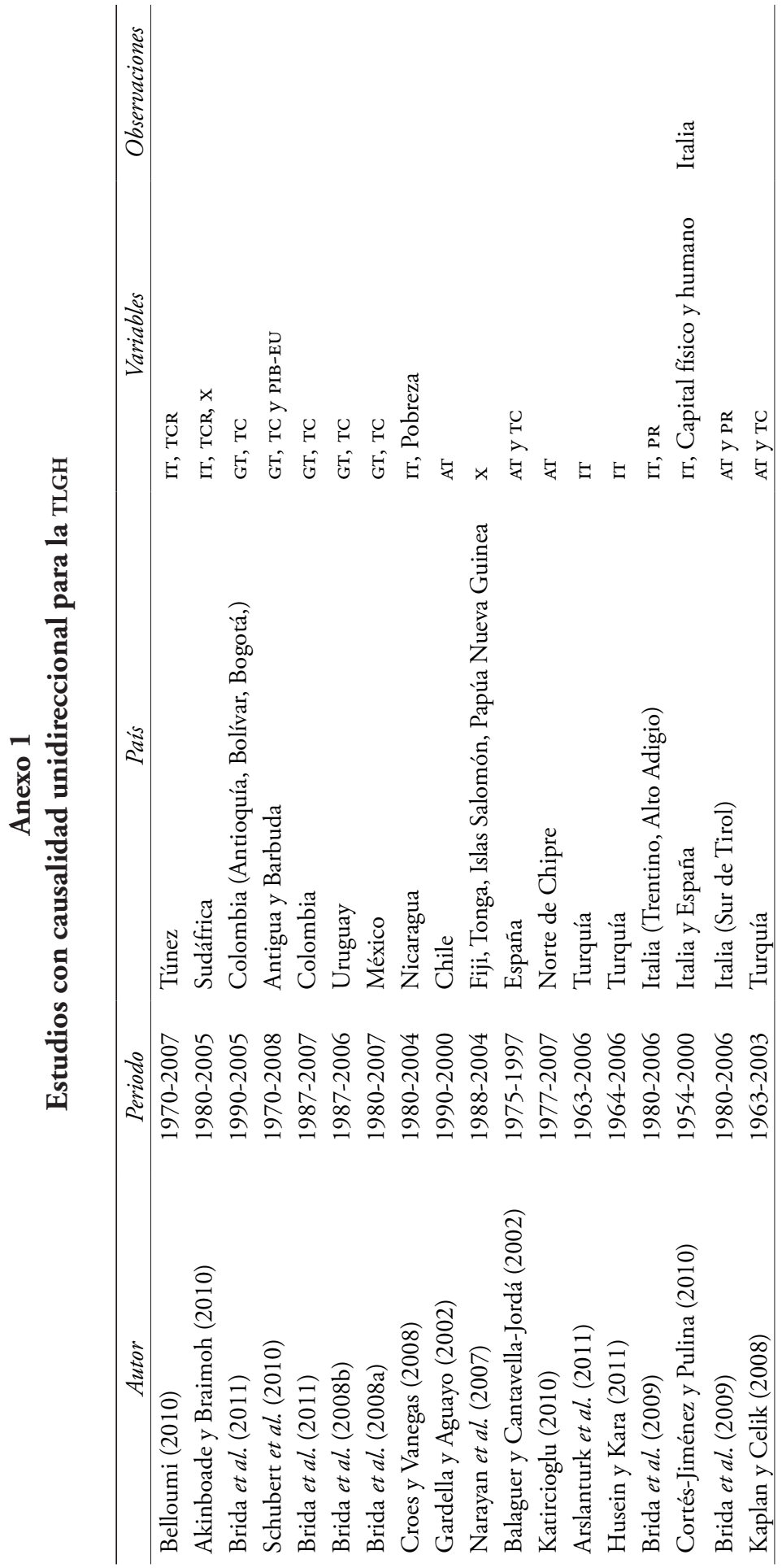




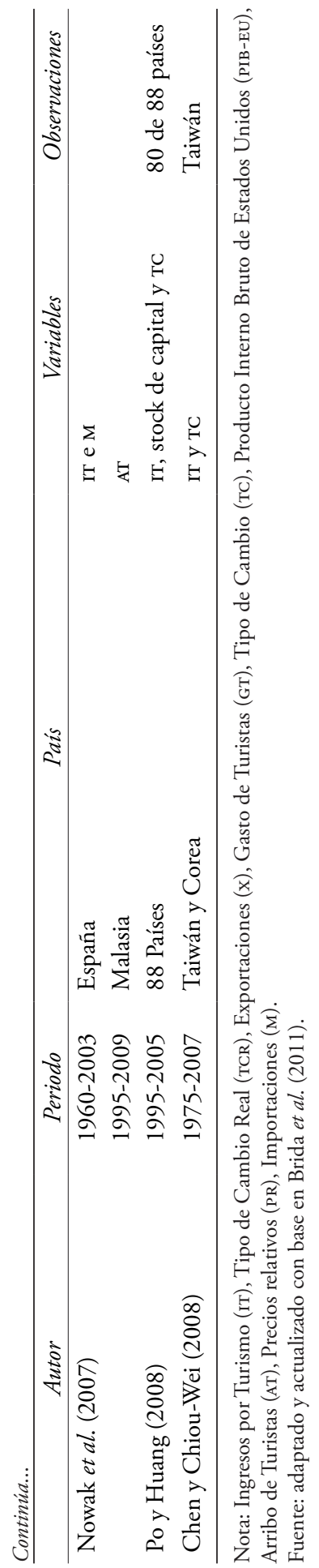




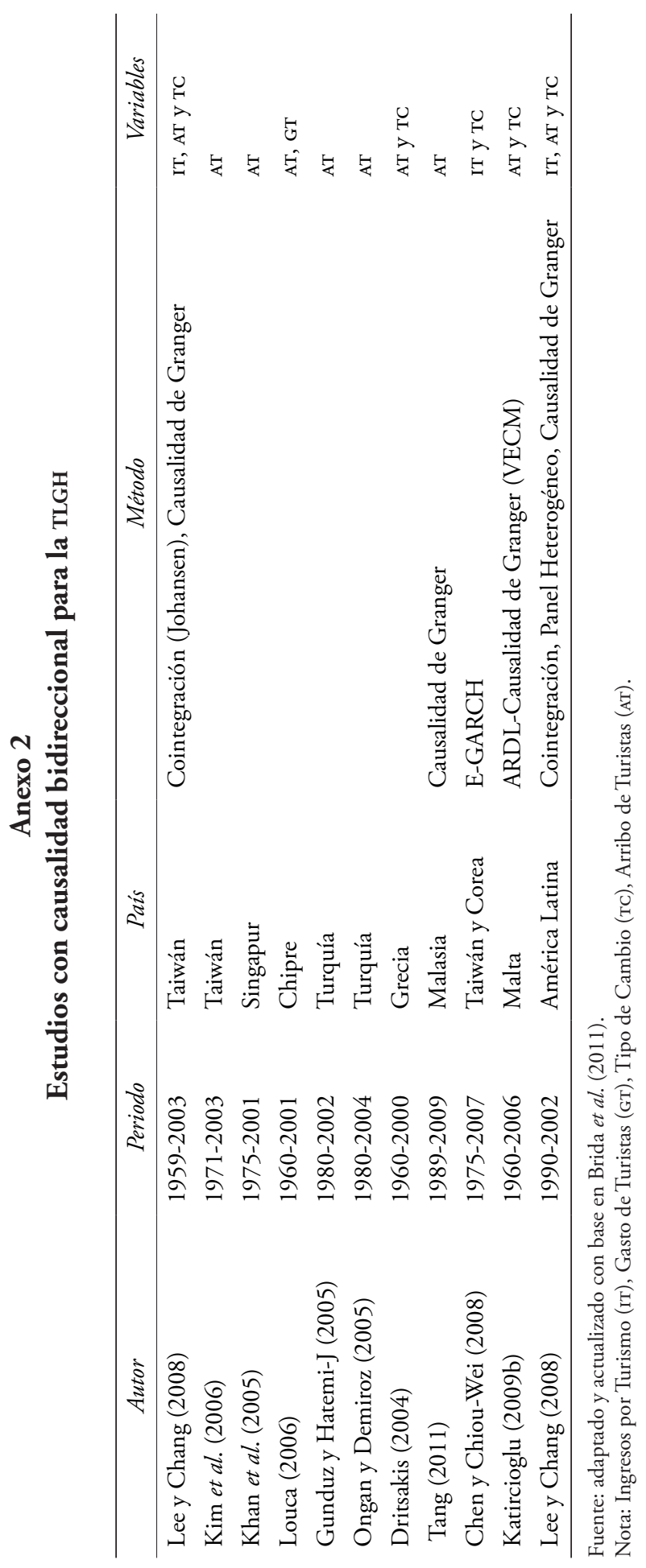




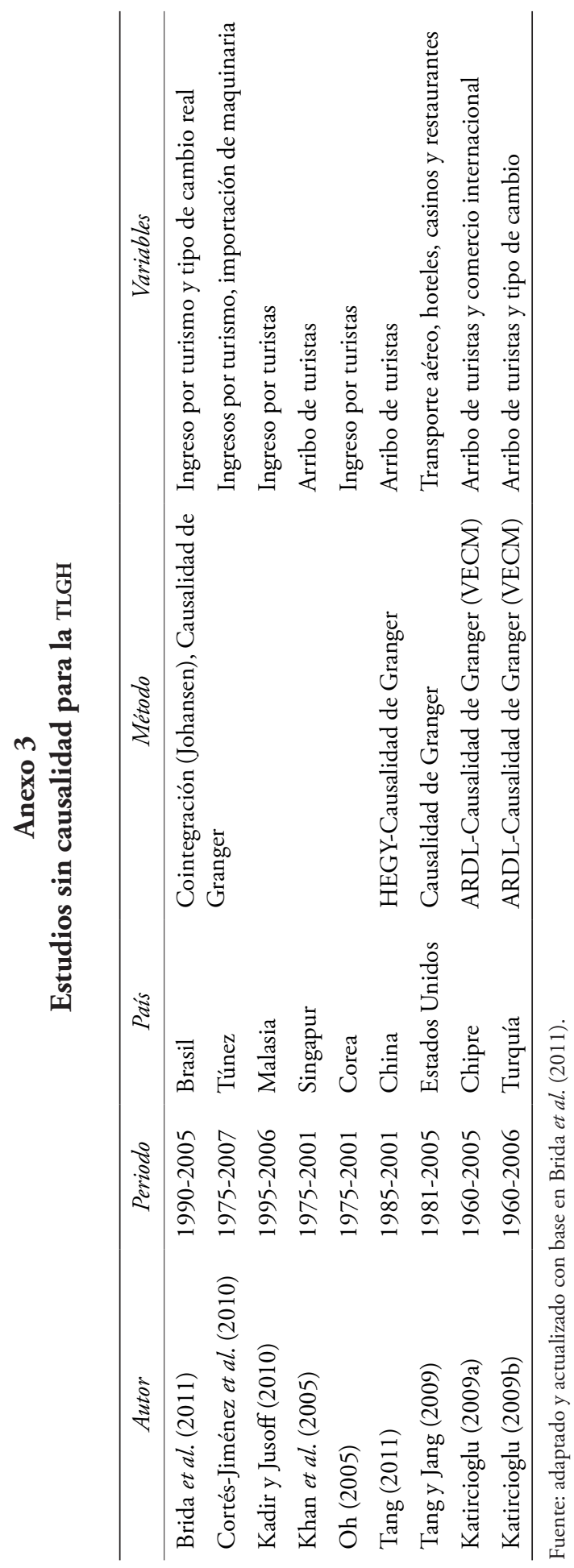


Anexo 4

Pruebas de normalidad para los residuos del MCE

\begin{tabular}{lrrrr}
\hline & \multicolumn{2}{c}{ Doornik-Hansen } & \multicolumn{2}{c}{ Lütkepohl } \\
\multicolumn{1}{c}{ Prueba } & Estadistico & Probabilidad & Estadistico & Probabilidad \\
\hline Conjunta & 9.9916 & 0.1250 & 10.2378 & 0.1150 \\
Asimetría & 5.3110 & 0.1504 & 5.1418 & 0.1617 \\
Curtosis & 4.6806 & 0.1967 & 5.0960 & 0.1649 \\
\hline
\end{tabular}

Fuente: elaboración propia.

\section{Anexo 5 \\ Diagnostico del MCE}

\begin{tabular}{lrr}
\hline Prueba de autocorrelación & \multicolumn{1}{c}{ Estadístico } & Probabilidad \\
\hline LM (1) & 9.5846 & 0.3851 \\
LM (2) & 9.0590 & 0.4318 \\
LM (3) & 12.1439 & 0.2053 \\
\hline
\end{tabular}

Fuente: elaboración propia. 


\section{Anexo 6 \\ Función impulso-respuesta}

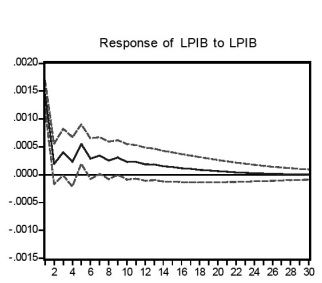

Response to Cholesky One S.D. Innovations \pm 2 S.E.
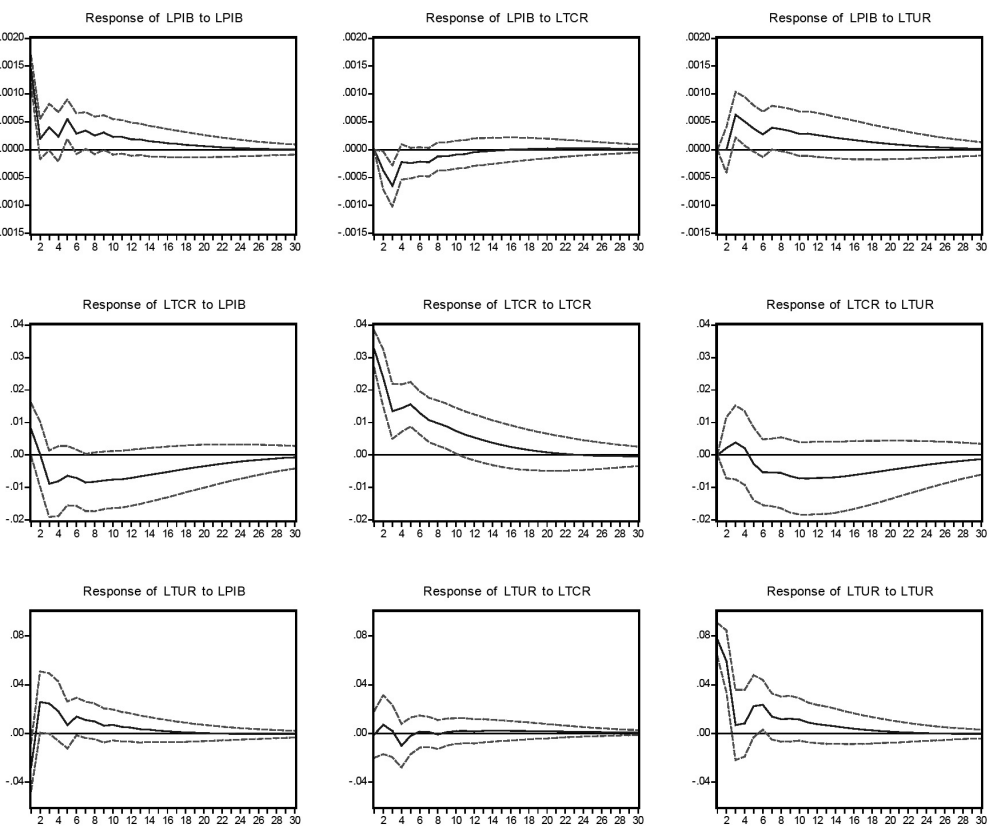

Fuente: elaboración propia.

Víctor Ángel Hernández-Trejo. Doctor en ciencias marinas y costeras, maestro en economía ambiental y de los recursos naturales y licenciado en economía por la Universidad Autónoma de Baja California Sur. Es miembro del Sistema Nacional de Investigadores. Actualmente funge como profesor-investigador en el Departamento Académico de Economía en la Universidad Autónoma de Baja California Sur. Sus líneas de investigación son economía ambiental, economía sectorial y economía aplicada; entre sus publicaciones más recientes se encuentran: "Economic valuation of water in a natural protected area of an emerging economy: recommendations for El Vizcaino Biosphere Reserve, Mexico", Interciencia, 38 (4), Asociación Interciencia, Caracas, pp. 245-252 (2013); "Mercado de derechos de agua para uso agrícola en el noroeste de México", Revista Mexicana de Ciencias Agrícolas, 4 (1), Instituto Nacional de Investigaciones Forestales, Agrícolas y Pecuarias, Quéretaro, pp. 63-75 (2013); "Economic benefits of the sport fishing in Los Cabos, Mexico: Is the relative abundase a determinant?", en F. D. Pineda y C. A. Brebia (eds.), Sustainable tourism V, WIT Press, Ashurst, pp. 165-172 (2013); "Beneficios económicos de los servicios recreativos provistos por la biodiversidad 
acuática del Parque Nacional Archipiélago Espíritu Santo”, Estudios Sociales, XX (40), Coordinación de Desarrollo Regional, Hermosillo, pp. 156-177 (2012).

Gerzaín Avilés-Polanco. Doctor en ciencias en el uso, manejo y preservación de los recursos naturales por el Centro de Investigaciones Biológicas del Noroeste, maestro en economía aplicada por el Colef y licenciado en economía por la Universidad Autónoma de Baja California Sur. Pertenece al Sistema Nacional de Investigadores. Actualmente funge como profesor-investigador en el Departamento Académico de Economía en la Universidad Autónoma de Baja California Sur. Sus líneas de investigación son economía ambiental, economía sectorial y economía aplicada. Entre sus publicaciones más recientes se encuentran: "Economic valuation of water in a natural protected area of an emerging economy: recommendations for El Vizcaino Biosphere Reserve, Mexico", Interciencia, 38 (4), Asociación Interciencia, Caracas, pp. 245-252 (2013); "Beneficios económicos de los servicios recreativos provistos por la biodiversidad acuática del Parque Nacional Archipiélago Espíritu Santo", Estudios Sociales, XX (40), Coordinación de Desarrollo Regional, Hermosillo, pp. 156-177 (2012); "Análisis socioeconómico de la pesquería de calamar gigante en Guaymas, Sonora", Economía, Sociedad y Territorio, XI (37), El Colegio Mexiquense, A. C., Zinacantepec, pp. 645-666 (2011); "Valoración económica del servicio hidrológico del acuífero de La Paz, BCs: Una valoración contingente del uso de agua municipal”, Frontera Norte, 22 (43), El Colegio de la Frontera Norte, Tijuana, pp. 103-128 (2010).

Gustavo Cruz-Chávez. Actualmente estudiante de doctorado en desarrollo económico y sectorial estratégico en la Universidad Popular Autónoma del Estado de Puebla. Actualmente funge como profesor-investigador en el Departamento Académico de Economía en la Universidad Autónoma de Baja California Sur. Sus líneas de investigación actuales son desarrollo económico, desarrollo sectorial y análisis económico de políticas públicas. Entre sus publicaciones más recientes se encuentran: Politicas de descentralización y gobierno local. El desarrollo turistico de Los Cabos, Universidad Autónoma de Baja California Sur-Gobierno del Estado de Baja California Sur (2006); Procesos territoriales en Baja California Sur: integración funcional y desigualdades regionales, Universidad Autónoma de Baja California Sur, La Paz (2006); "Panorama agropecuario en Baja California Sur", Diagnóstico estratégico de Baja California Sur, Universidad Autónoma de Baja California Sur-Secretaría de Educación Pública, La Paz, pp. 183-200 (2003). 
Ramón Valdivia-Alcalá. Doctor en economía por el Colegio de Posgraduados, maestro en economía agrícola y recursos naturales y licenciado en economía agrícola por la Universidad Autónoma Chapingo. Pertenece al Sistema Nacional de Investigadores; actualmente es profesor definitivo en la División de Ciencias Económico-Administrativas de la Universidad Autónoma Chapingo, sus líneas de investigación son economía de la empresa y economía de los recursos naturales. Entre sus publicaciones más recientes se encuentran, en coautoría: "Valoración económica del reciclaje de residuos urbanos", Revista Chapingo. Serie Ciencias Forestales y del Ambiente, 18 (3), Universidad Autónoma Chapingo, Texcoco, pp. 435-448 (2012); "Cobertura óptima en el mercado de futuros bajo riesgo de precio y rendimiento", Revista Mexicana de Ciencias Agrícolas, 3 (6), Instituto Nacional de Investigaciones Forestales, Agrícolas y Pecuarias, Metepec, pp. 1275-1284 (2012); "Valoración económica del agua en el sector industrial”, Terra Latinoamericana, 29 (4), Universidad Autónoma Chapingo, Texcoco, pp. 459-466 (2011); "Valoración económica de los beneficios de un programa de recuperación y conservación en el Parque Nacional Molino de Flores, México", Revista Chapingo. Serie Ciencias Forestales y del Ambiente, 17 (2), Universidad Autónoma Chapingo, Texcoco, pp. 231-244 (2011). 